doit, dans une certaine mesure, devenir proportionnelle à la production, cela ne peut se produire que conjointement avec le développement d'une confiance générale et complète dans la propreté et la salubrité de la fourniture de lait tout entière. A cet effet, il doit être spécifié que, dans des limites bien déterminées, non seulement la production " catégorisée " et " accréditée " doit répondre aux conditions spécialement établies, mais eneore que, partout et toujours, tout lait doit être propre et de bonne qualité, et que lors de la livraison au consommateur la salubrité de tout lait doit être absolue et non pas relative. Ces exigences auraient dû être établies depuis longtemps ; actuellement, sans aucun obstacle, sans aucun empêehement, on distribue tous les jours de grandes quantités de lait, qui ne présentent pas la moindre garantie hygiénique. Si la confiance est la condition essentielle de la prospérité, peut-il exister quelque espoir pour l'industrie, aussi longtemps que les conditions actuelles ne seront pas modifiées?

$$
*^{*} *
$$

En terminant cet exposé, nous tenons à remercier, sans distinction aucune, tous les membres du personnel des laboratoires des "United Dairies" pour le concours enthousiaste qu'ils nous ont apporté au cours des recherches qui ont précédé la publication de ce Mémoire. Ces recherches spéciales eurent une durée d'environ trois ans. Au risque de froisser leur modestie, nous tenons à mentionner l'assistance, en tout temps, de MM. E. B. Anderson, L, J. Meanwell et Leslie J. Walker.

\title{
REVUE
}

\section{LA PRODUCTION MONDIALE DE CASÉINE}

\author{
par \\ G. GENIN \\ Ingénieur E. P. C.
}

La demande mondiale de caséine, quelle que soit sa qualité, s'est développée dans de grandes proportions depuis déjà quelques mois. Il en est résulté naturellement une augmentation de la production et une élévation des prix. Ce développement de la consommation provient d'un afflux de demandes de la part de l'industrie des matières plastiques, des colles, des détrempes, du papier couché, des insecticides, de l'industrie textile et naturellement des produits alimentaires de régime et des produits médicinaux.

La production mondiale de caséine (caséine à la présure et caséine 
obtenue par acidification naturelle) était de l'ordre de 60.000 tonnes en 1932 ; on estime qu'en 1936 elle a atteint 70.000 tonnes. Il n'y a d'ailleurs que quelques pays à produire en grande quantité et à exporter de la caséine: l'Argentine, la France et les Etats-Unis, ces deux premiers pays étant encore aujourd'hui des pays agricoles.

La production française s'élève en moyenne à 16.000 tonnes par an, localisée principalement dans la Charente, où existe une coopérative de producteurs très active et où l'on produit de la caséine de présure de très bonne qualité. La production de l'Argentine oscille autour de 18.000 tonnes et se compose principalement de caséine obtenue par acidification naturelle. Celle des Etats-Unis atteint également 18.000 tonnes et se compose des caséines des deux types.

L'Allemagne est dans l'obligation d'importer la caséine nécessaire à ses besoins, qui représentent $30 \%$ des exportations totales mondiales. Le gouvernement s'oppose en effet à ce que le petit-lait soit utilisé pour la préparation de la caséine, tant que les cours de ce produit ne seront pas plus rémunérateurs. C'est la raison pour laquelle la production allemande ne dépasse pas 300 tonnes, soit seulement le cinquième de la production australienne.

Depuis quelques années, différents pays se sont efforcés de eréer sur leur propre sol une industrie de la easéine en dépit des variations de cours que subit ce produit. En 1929 par exemple, le cours le plus élevé à Londres avait été de $63 £$ par tonne (long ton). Au mois d'octobre 1935 , les cours se rapprochaient de $37 £$ atteignant presque les chiffres les plus bas enregistrés en 1931, au pire moment de la crise mondiale et qui étaient de $20 £$.

Depuis le début de 1936, les prix sont par contre en hausse régulière et ont atteint $49 £$ en octobre. Au début de novembre 1936, des bruits de "manque de caséine" ont commencé à apparaître sur les marchés et les Etats-Unis ont envoyé de très importants ordres en France.

Un autre débouché très important s'est ouvert à la caséine, eelui de la fabrication de la laine artificielle dite "Lanital ». Jusqu'ici, l'Italie était le seul pays où cette fabrication était assurée par la Snia Viscosa de Milan, mais les producteurs français de caséine, tenant compte de la dévaluation du franc qui a abaissé relativement aux prix mondiaux les prix de la caséine fabriquée en France, auraient à leur tour l'intention de s'intéresser à la fabrication de la laine synthétique.

Les récentes recherches entreprises sur Ia caséine, au point de vue technique comme au point de vue chimique, ont permis de faire de cette substance une matière première du plus haut intérêt. II est indiscutable que nos connaissances sur la chimie de la caséine 
sont encore très obscures; cependant M. C. E. Rowe, au cours d'une récente conférence, a donné la composition pondérale de la caséine commerciale : carbone, $53,3 \%$; azote, $15,85 \%$; hydrogène, $7,1 \%$; phosphore, $0,85 \%$, mais le point de vue purement chimique de la question est plutôt regardé comme secondaire, l'étude physicochimique de la caséine paraissant plus pleine de promesses, en particulier pour permettre d'apporter une plus grande uniformité dans les livraisons de caséine.

Depuis que les producteurs de tourteaux de soja ont proposé de remplacer la caséine lactique par une caséine végétale, les produeteurs de produit animal se sont en général associés pour améliorer leur industrie et tendre à la production de caséine lactique d'une qualité parfaite classée en différentes qualités suivant ses applications.

Les recherches entreprises dans ce but ont surtout porté sur la caséine obtenue par acidification naturelle ou par addition d'acide, caséine que l'on estime la plus intéressante au point de vue industriel, par suite de sa meilleure solubilité et de la propriété qu'elle a de donner des solutions moins visqueuses. Cette caséine est également supérieure, au point de vue propriétés mécaniques, à la caséine à la présure, cette dernière étant surtout utilisée par l'industrie des matières plastiques.

Nous rappellerons que la caséine française à la présure (les Américains l'appellent souvent à tort paracaséine) est plus jaune que les produits provenant de l'Argentine ou de la Nouvelle-Zélande.

L'augmentation mondiale de la consommation mondiale de caséine est généralement due à un développement de ses applications dans les diverses industries déjà intéressées à ce produit, développement qui résulte en grande partie des progrès réalisés dans les laboratoires industriels. C'est ainsi que par des études prolongées, on est parvenu à prolonger la "vie» des colles à la caséine et à étendre leur application. Par vie d'une colle à la caséine, on entend la durée qui suit immédiatement sa préparation et pendant laquelle on peut l'utiliser avant qu'elle devienne trop visqueuse. Ordinairement la vie d'une colle à la caséine est courte, 30 minutes environ. Malgré cet inconvénient, les colles à la caséine étaient préférées aux autres colles végétales ou animales, par suite de leur résistance à l'humidité et à la chaleur. Dans la fabrication du bois contre-plaqué en particulier, ces colles étaient très employées, du fait de la possibilité de les employer à froid en évitant ainsi toute déformation du bois. Toutefois le fait d'avoir pu prolonger la "vie " d'une colle à la caséine a permis son emploi dans les grandes fabriques de meubles. On a constaté qu'en mélangeant du silicate de sodium avec de la chaux à la place de soude caustique et en ajoutant 
ce mélange à la caséine, on peut prolonger la durée de la vie de la colle jusqu'à 24 heures. De plus on peut encore améliorer les propriétés adhésives de la colle en lui ajoutant de l'acide tannique et d'autres dérivés des tanins.

Des recherches de laboratoire ont également permis l'emploi des colles à la caséine dans un domaine nouveau, celui du collage du bois sur les métaux dans l'industrie automobile, du collage du caoutchouc et du linoléum sur le béton constituant les planchers des bâtiments.

Les colles à la caséine sont également de plus en plus utilisées pour la fabrication des étiquettes gommées, elles remplacent dans ce cas la dextrine et pour cette application particulière on a conseillé un mélange de caséine, de borax et de résinate de sodium, ce mélange évitant tout décollage des étiquettes sous l'action de la chaleur ou de l'humidité.

Les excellentes propriétés de la caséine ont également favorisé le développement de la fabrication du papier couché et c'est ainsi qu'aux Etats-Unis, on estime que les deux tiers de la consommation de caséine de ce pays sont réservés à l'industrie du papier. Même développement de la demande dans la fabrication des peintures à l'eau, depuis qu'on sait préparer ces peintures sous la forme de poudre et qu'on a pu les rendre lavables après leur application.

\section{BIBLIOGRAPHIE ANALYTIQUE}

\section{LES LIVRES}

SaInt-Cyr (J.). - Laits pauvres. Laits écrémés. Répression de l'écrémage. Modification à y apporter. 1 brochure $16 \times 25,97$ pages, nombreux tableaux et courbes. Editions du Fleuve, Lyon, 42, quai Gailleton, 1937.

Dans ce travail bien présenté, mon camarade SAINT-CYR reprend l'argumentation qu'il avait déjà développée, en collaboration avec BoUDET, dans une communication récente à la Société des Sciences vétérinaires de Lyon. Il s'agit, en fait, de faire cesser les injustices flagrantes qui résultent d'après l'A. de l'application des méthodes actuelles de répression de l'écrémage. SAINT-CYR, qui appartient à une famille terrienne, qui vit au milieu des agriculteurs, qui les comprend et qui les aime, a vu les lamentables ravages que peut causer dans une famille honorable l'erreur judiciaire si facile à commettre en matière d'écrémage. En honnête homme, il a cherché à s'informer et j'ai eu le plaisir au début de ses recherches de l'aider et de l'orienter ; nous avons bien souvent agité ensemblẹ le problème, c'est-à-dire que je partage absolument les idées qu'il expose dans ses chapitres IV et V (Modifications à apporter aux méthodes de répression de l'écrémage). 